\title{
Richesse de la pharmacopée malinké : rôle médicinal de l'arbre a Khossanto : (Kédougou, Sénégal oriental)
}

\author{
Oumar Ndao GNING ${ }^{\text {* }}$, Oumar SARR ${ }^{1}$, Leonard Elie AKPO1 \\ 1 UCAD/FST (UCAD-PPZS), Laboratoire d'Écologie et d'Eco-hydrologie, BP n 5005 Dakar-Fann, Sénégal) ; \\ ${ }^{*}$ Correspondant : OumarNdao GNING, Email : gningoumarndao@yahoo.fr
}

Original submitted in on $3^{\text {rd }}$ December 2013 Published online at www.m.elewa.org on $28^{\text {th }}$ February 2014. http://dx.doi.org/10.4314/jab.v74i1.11

\begin{abstract}
RESUME
Objectif : Apprécier la richesse de la pharmacopée malinké et l'importance des ligneux dans la santé des populations de Khossanto.

Méthodologie et résultats : Des entretiens ethnobotaniques ont été menés dans la communauté rurale de Khossanto. La variabilité des usages et leurs intérêts phytothérapiques ont été appréciés à travers les fréquences moyennes de citations, les facteurs de consensus informateurs, les niveaux de fidélités des espèces et la description des modes d'utilisation. Les usages médicinaux des ligneux font l'objet d'un assez large consensus (81\%). 76\% des enquêtés reconnaissent des vertus thérapeutiques à $87 \%$ des espèces ligneuses utiles de la localité. Elles traitent une quarantaine d'affections, des plus banales aux plus préoccupantes. Six espèces sont plus fréquemment indiquées. Presque toutes les parties de l'arbre sont utilisées. Plusieurs modes préparatoires et d'administration sont décrits.

Conclusion et application des résultats : L'arbre joue un rôle essentiel dans la santé des populations de Khossanto. Vitellaria paradoxa et Borassus aethiopum s'avèrent être les espèces ligneuses les plus utilisées pour la santé des populations de Khossanto. Les écorces et les feuilles sont les parties des ligneux les plus utilisées. Ces parties utilisées semblent déterminer les modes préparatoires, dont les plus fréquents sont la macération et l'infusion. Et ces modes préparatoires seraient eux aussi corrélés aux modes d'administration, dont les plus communs sont la boisson et l'utilisation pour le bain. Par ailleurs, pour leurs forts niveaux de fidélité pour des traitements spécifiques, la recherche d'éléments bioactifs contre des affections psycho-émotionnelles, le paludisme, l'asthénie, les nausées/vomissements et la fièvre jaune pourraient être envisagée respectivement chez Bambusa vulgaris, Saba senegalensis, Vitellaria paradoxa, Ziziphus mauritiana et Borassus aethiopum.
\end{abstract}

Mots clés : Ligneux-arbre - pharmacopée- phytothérapie - Sénégal - Kédougou.

\section{ABSTRACT}

Richness of Malinké pharmacopoeia: role of medicinal trees in Khossanto (Kédougou, eastern Senegal)

Objective: To assess the richness of the Malinke pharmacopoeia and the importance of medicinal trees in the health of the Khossanto population.

Methodology and results: Ethno botanical interviews were undertaken with populations of Khossanto rural community. The usages variability, theirs interests for populations and the local knowledge on the tree were 
appreciated through the average frequency of indication, consensus informant factors, fidelity levels of the species and usage mode description. The medicinal uses of ligneous were subject to a broad consensus (81\%). $76 \%$ of respondents recognize therapeutic value $87 \%$ of useful ligneous species of the locality. They allow to dealing with forty ailments, from most mundane to most worrying. Six species are most frequently indicated. Almost all parts of the tree are used. Several methods of preparation and administration are described.

Conclusion and application of findings: Trees play a vital role in the health of Khossanto populations. Vitellaria paradoxa and Borassus aethiopum seem to be the most used ligneous species for Population Health. The bark and leaves are the most used parts of the tree. These used parts seem to determine the preparatory methods, which the most common are the maceration and the infusion. In addition, these preparatory methods seem to be also correlated with the administration modes, which the most common are the drink and use for bathing. In addition, for their high levels of fidelity to specific treatments, research of bioactive components against psycho-emotional disorders, malaria, fatigue, nausea/vomiting and yellow fever could be considered respectively with Bambusa vulgaris, Saba senegalensis, Vitellaria paradoxa, Ziziphus mauritiana and Borassus aethiopum.

Key words: Tree, pharmacopoeia, phototherapy, Senegal, Kédougou.

\section{INTRODUCTION}

En milieu rural, l'arbre joue un rôle essentiel dans la vie des populations. En effet, dans les parcours communautaires les ligneux sont utilisés pour la santé $(90 \%)$, dans l'affouragement du bétail $(80 \%)$, comme source d'énergie domestique $(71 \%)$, dans l'alimentation humaine $(61 \%)$, en construction (16\%), en artisanat (14\%) (Gning et al., 2013). La phytothérapie est une pratique millénaire qui utilise des savoirs empiriques; elle est sans conteste la médecine la plus vieille du monde. Toutes les sociétés, antiques ou modernes, ont une médecine des plantes (VeneyTherapies, 2013). Ce rôle central des plantes dans la santé des populations, confirme la dépendance "dans certains pays d'Asie et d'Afrique de la population (80\%) de la médecine

\section{MATERIELS ET METHODES}

La zone d'étude: L'étude a été menée dans la communauté rurale de Khossanto, qui relève de l'arrondissement de Bembou, du département de Saraya, de la région de Kédougou (Figure 1). Située à l'extrême Sud - Est du Sénégal, elle est limitée au Nord et à l'Ouest par la région de Tambacounda, à l'Est par la République du Mali et au Sud par la République de Guinée Conakry).

Caractéristiques physiques : Le climat de Kédougou est tropical de type subhumide caractérisé par l'harmattan, chaud et sec le jour, et frais la nuit en raison de l'influence de la mousson. Les pluviométries annuelles ont varié, à Saraya, entre 400 et $1600 \mathrm{~mm}$, traditionnelle pour les soins de santé primaires. Dans de nombreux pays en développement, $70 \%$ à $80 \%$ de la population a eu recours à une forme ou une autre de médecine alternative ou complémentaire" (OMS, 2012). Dans la communauté rurale de Khossanto, peu équipée en infrastructures sanitaires, la phytothérapie est assez répandue. De nombreuses espèces sont utilisées pour soigner les populations sous diverses formes. Ainsi dans ce travail, nous avons cherché à donner un aperçu de la pharmacopée malinké et établir l'importance des ligneux dans le traitement des différentes affections des populations.

avec une moyenne interannuelle globale de $1049 \mathrm{~mm}$ (1950-2009). Depuis 1968, la zone connaît une péjoration climatique, avec 26 années $(83,87 \%)$ déficitaires $(P m=972 m m)$. Le bilan climatique (figure 3 ) montre que les pluies sont notées sur 6 mois (mai octobre), avec 5 mois d'humidité effective (mi-mai à la mi-octobre). Le relief de la région est formé de vastes étendues aplanies dominées localement par des buttes cuirassées. (Camus, Debuisson, 1964). Elle dispose d'un réseau hydrographique, tributaire de trois grands cours d'eau pérennes (le Sénégal, la Falémé et la Gambie), de leurs affluents et d'un réseau très dense 
de marigots dont les tracés sont excessivement sinueux (Diouf, 1999).

La population et ses activités: La population de Kédougou, estimée à 125000 habitants, est répartie entre Malinkés, Bambaras, Sarakholés, Pulaars, Wolofs, Sérères, Diolas, Maures, Bediks, etc. La population est relativement jeune $(56 \%$ de moins de 25 ans) et les femmes représentent environ $52 \%$. La population de la communauté rurale de Khossanto, estimée à 12500 habitants dont ( $80 \%$ de Malinkés), reste essentiellement agropastorale et associe ainsi l'agriculture à l'élevage.

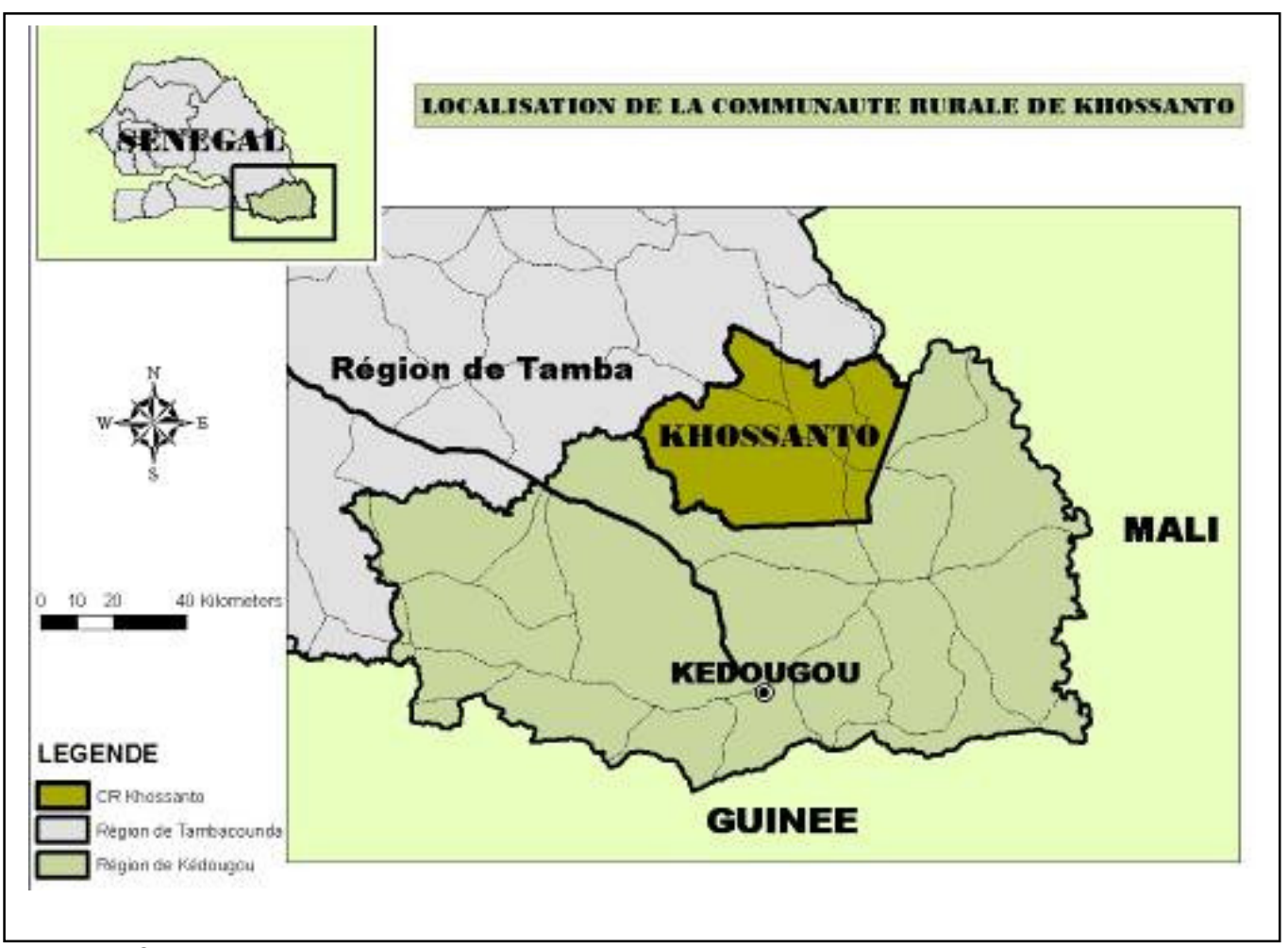

Figure 1: Carte de situation de la zone d'étude.

Méthodes utilisées: Sur la base des enquêtes réalisées dans le cadre de l'étude sur la valeur socioéconomique de l'arbre en milieu malinké (Gning et al., 2013), nous nous sommes intéressés particulièrement aux usages de ces plantes pour les soins des populations. Le dépouillement des questionnaires a d'abord été manuel. La saisie des réponses a été faite avec le logiciel Sphinx Plus ${ }^{2}$ Développement. Sphinx permet de génèrer directement les résultats en fonction des variables de saisie en utilisant les techniques d'analyses uni-variées ou bi-variées. Certaines analyses statistiques ont nécessité en plus l'usage du tableur Excel. La richesse floristique de la pharmacopée malinké a été établie avec la répartition des espèces phytothérapiques à travers leurs genres et familles botaniques. L'importance phytothérapique de chaque espèce ligneuse a été appréciée en calculant les
Fréquences Moyennes de Citations $(F M C=$ Nombre de citations d'une espèce $\times 100 /$ Nombre total de répondants) et à leurs Niveaux de Fidélité (Fidelity level: $\boldsymbol{F L}$ de Cheikhyoussef et al., 2011) à la phytothérapie. Le niveau de fidélité ( $\mathrm{FL}$ ) apprécie l'intensité de la relation que les populations établissent entre une espèce et son (ses) rôle(s). II est ainsi calculé : $\mathrm{FL}=\mathrm{N}_{\mathrm{p}} / \mathrm{N} \times 100$. Où $\mathrm{N}_{\mathrm{p}}$ désigne le nombre d'indication d'une espèce en phytothérapie et $\mathrm{N}$ le nombre total d'indication de l'espèce pour tous les usages. Le niveau de fidélité est élevé quand une espèce est systématiquement inféodée à l'usage thérapeutique ou à un nombre réduit d'usages. Par contre, si une espèce est indiquée à la fois pour plusieurs usages, son niveau de fidélité sera faible. Aussi, la variabilité des usages phytothérapiques a été étudiée à travers la description des affections traitées, des parties utilisées, des modes de préparation et d'administration pour chaque espèce ligneuse 
phytothérapique. En fin, la répartition des usages phytothérapiques en catégories, ainsi que le Facteur de Consensus Informateur (Informant Consensus Factor $\left(\boldsymbol{F}_{i c}\right)$ défini par Heinrich et al., 1998a, in Canales et al., 2005) nous ont permis d'apprécier la diversité et la consistance indications phytothérapiques. Le Facteur de Consensus Informateur $\left(F_{i c}\right)$, toujours compris entre 0 et 1 , est élevé quand une seule ou un nombre réduit d'espèces sont citées par une grande proportion

\section{RESULTATS}

Richesse de la pharmacopée malinké : Dans la flore ligneuse de la zone d'étude, nous avons inventorié plusieurs groupes d'espèces selon les usages. $76 \%$ des enquêtés reconnaissent des vertus thérapeutiques à $87 \%$ des espèces ligneuses utiles de la localité. Ces espèces utiles dans le traitement des affections sont dans le tableau 1. II apparait qu'à Khossanto, 40 espèces ligneuses (des 46 utilisées par les populations) ont un rôle thérapeutique. Ces espèces ligneuses phytothérapiques relèvent de 37 genres et de21 familles. D'un point de vue taxonomique, les familles les plus importantes sont Caesalpiniaceae d'informateurs pour type de traitement spécifique. A l'inverse, sa valeur sera d'autant plus proche de 0 qu'il ait une grande diversité d'espèces citées pour un même traitement. Le $F_{i c}$ est calculé à partir de la formule suivante: $F_{i c}=N_{u r}-N_{t} /\left(N_{u r}-1\right)$. Où $N_{u r}$ (userreports number) est le nombre d'indication pour untraitement donné et $\mathbf{N}_{t}$ (number of taxa) le nombre d'espèces impliquées dans cette même catégorie d'usage.

riche de 5 espèces et d'autant de genres; Combretaceae avec 5 espèces relevant de 3 genres; et les Rubiaceae forte de 4 espèces appartenant chacune à un genre distinct.

Les usages phytothérapiques: Les espèces ligneuses utilisées en phytothérapie ont fait l'objet de 201 expressions d'usages, dont les espèces impliquées, les parties utilisées, les affections ou maux traités et les modes de préparation et d'administration sont décrits dans le tableau $n^{\circ} 2$. 
$\begin{aligned} & \text { Oumar et al. J. Appl. Biosci. 2014. Richesse de la pharmacopée malinké : rôle médicinal de l'arbre a } \\ & \text { khossanto, Sénégal }\end{aligned}$

Tableau 1 : Espèces phytothérapiques parmi les espèces ligneuses utiles de Khossanto.

\begin{tabular}{|c|c|c|c|c|c|c|c|}
\hline Familles & Genres & Espèces & $\begin{array}{c}\text { Esp. } \\
\text { Phyt } \\
\text {. }\end{array}$ & Familles & Genres & Espèces & $\begin{array}{c}\text { Esp. } \\
\text { Phyt } \\
.\end{array}$ \\
\hline \multirow{2}{*}{ Anacardiaceae } & Lannea & L. acida & + & Fabaceae & Afrormosia & A.laxiflora & + \\
\hline & Spondias & S. mombin & + & Euphorbiaceae & Hymenocardia & H.acida & + \\
\hline \multirow{2}{*}{ Annonaceae } & Annona & A.senegalensis & + & Loganiaceae & Strychnos & S.spinosa & - \\
\hline & Hexalobus & H.monopetalus & + & \multirow{2}{*}{ Méliaceae } & Khaya & K.senegalensis & + \\
\hline Apocynaceae & Saba & S.senegalensis & + & & Pseudocedrela & P.kotschyi & + \\
\hline Arecaceae & Borassus & B.aethiopum & + & \multirow{4}{*}{ Mimosaceae } & \multirow{2}{*}{ Acacia } & A.dudgeoni & + \\
\hline Balanitaceae & Balanites & B.aegyptiaca & + & & & A.seyal & - \\
\hline Bignoniaceae & $\begin{array}{l}\text { Stereospermu } \\
m\end{array}$ & S.kunthianum & + & & Parkia & P.biglobosa & + \\
\hline Bombacaceae & Adansonia & A.digitata & + & & Prosopis & P.africana & + \\
\hline Boraginaceae & Cordia & C.myxa & + & \multirow[b]{2}{*}{ Moraceae } & \multirow[b]{2}{*}{ Ficus } & F.dicranostyla & + \\
\hline \multirow{6}{*}{ Caesalpiniaceae } & Afzelia & A.africana & - & & & $\begin{array}{l}\text { F.gnaphalocarp } \\
a\end{array}$ & + \\
\hline & Cassia & C.sieberiana & + & Olacaceae & Ximenia & X.americana & + \\
\hline & Cordyla & C.pinnata & + & Papilionaceae & Pterocarpus & P.erinaceus & + \\
\hline & Detarium & D.microcarpum & + & Poaceae & Bambusa & B.vulgaris & + \\
\hline & Piliostigma & P.reticulatum & + & Rhamnaceae & Ziziphus & Z.mauritiana & + \\
\hline & Tamarindus & T. indica & + & \multirow{4}{*}{ Rubiaceae } & Crossopteryx & C.febrifuga & + \\
\hline \multirow{6}{*}{ Combretaceae } & Anogeissus & A.leiocarpus & - & & Gardenia & G.erubescens & + \\
\hline & \multirow{2}{*}{ Combretum } & C.glutinosum & + & & Mitragyna & M.inermis & + \\
\hline & & C.micranthum & + & & Nauclea & N.latifolia & + \\
\hline & Guiera & $\begin{array}{l}\text { G.senegalensi } \\
s\end{array}$ & + & Sapotaceae & Vitellaria & V.paradoxa & + \\
\hline & \multirow{2}{*}{ Terminalia } & T.avicennoides & + & $\begin{array}{l}\text { Simaroubacea } \\
\text { e }\end{array}$ & Hannoa & H.undulata & - \\
\hline & & T.macroptera & + & Tiliaceae & Grewia & G.bicolor & - \\
\hline Ebenaceae & Diospyros & D.mespiliformis & + & Verbénaceae & Vitex & V.madiensis & + \\
\hline
\end{tabular}

Esp. Phyt. = L'espèce a-t-elle un rôle phytothérapique ? ; "+" = Oui ; "-" = Non. 
Tableau 2 : Fréquence Moyenne de Citation (FMC), Niveau de Fidélité (FL), affections ou maux traitées, parties utilisées et mode d'emploi des espèces ligneuses en phytothérapie.

\begin{tabular}{|c|c|c|c|c|}
\hline ESPECES UTILES & $\begin{array}{l}\text { FMC } \\
(\%)\end{array}$ & $\mathrm{FL}(\%)$ & AFFECTIONS OU MAUX TRAITEES & PARTIES UTILISEES ET MODE D'EMPLOI \\
\hline \multirow{8}{*}{ Vitellaria paradoxa } & \multirow{8}{*}{31} & \multirow{8}{*}{20} & Rhume & Inhalation du beurre de Karité \\
\hline & & & Tuberculose, Toux & Macération aqueuse des feuilles et/ou de l'écorce pour boisson \\
\hline & & & Douleurs articulaires, Fatigue, Paresse & Massage corporel avec du beurre de Karité \\
\hline & & & Maladies infantiles & Massage corporel avec du beurre de Karité \\
\hline & & & Gale, Démangeaisons, Boutons & Massage corporel avec du beurre de Karité \\
\hline & & & Fièvre jaune & Macération aqueuse de l'écorce pour boisson \\
\hline & & & Crises épileptiques & $\begin{array}{l}\text { Macération aqueuse de la poudre des feuilles tombées à l'envers } \\
\text { pour bain corporel }\end{array}$ \\
\hline & & & Douleurs des dents & Macération aqueuse des feuilles pour bain corporel \\
\hline \multirow[t]{2}{*}{ Borassus aethiopum } & \multirow[t]{2}{*}{18} & \multirow[t]{2}{*}{11} & Fièvre jaune & $\begin{array}{l}\text { Liquide du fruit non mur pour boisson ou consommation directe du } \\
\text { sibinoxo (jeunes pousses) }\end{array}$ \\
\hline & & & Blennorragie "Chaude pisse" & Consommation directe des fruits \\
\hline \multirow{7}{*}{ Cordyla pinnata } & \multirow{7}{*}{15} & \multirow{7}{*}{8} & Parasitose & Infusion aqueuse de l'écorce pour boisson \\
\hline & & & Toux & Infusion aqueusede l'écorce pour boisson \\
\hline & & & Vomissement & Infusion aqueuse de l'écorce pour boisson \\
\hline & & & Faiblesse et douleurs chez la femme après accouchement & $\begin{array}{l}\text { Macération aqueuse de l'écorce pour boisson seule ou avec de la } \\
\text { bouillie de riz }\end{array}$ \\
\hline & & & Diarrhée & $\begin{array}{l}\text { Macération aqueuse de l'écorce pour boisson seule ou avec de la } \\
\text { bouillie de riz }\end{array}$ \\
\hline & & & Maux de ventre & $\begin{array}{l}\text { Macération aqueuse de l'écorce pour boisson seule ou avec de la } \\
\text { bouillie de riz }\end{array}$ \\
\hline & & & Fièvre jaune & $\begin{array}{l}\text { Macération aqueuse de l'écorce pour boisson, en association } \\
\text { avec le sibinoxo du Borassus aethiopum }\end{array}$ \\
\hline \multirow[t]{2}{*}{ Ziziphus mauritiana } & \multirow{2}{*}{15} & \multirow{2}{*}{14} & Toux, Nausée et Vomissement & Macération aqueuse de l'écorce pour boisson \\
\hline & & & Diarrhée & Macération aqueuse de l'écorce pour boisson \\
\hline
\end{tabular}




\begin{tabular}{|c|c|c|c|c|}
\hline ESPECES UTILES & $\begin{array}{l}\text { FMC } \\
(\%)\end{array}$ & $\mathrm{FL}(\%)$ & AFFECTIONS OU MAUX TRAITEES & PARTIES UTILISEES ET MODE D'EMPLOI \\
\hline & & & Maux de ventre & Macération aqueuse de l'écorce pour boisson \\
\hline & & & Blessures & Poudre de l'écorce et des feuilles à mettre dessus \\
\hline \multirow{5}{*}{ Saba senegalensis } & \multirow{5}{*}{12} & \multirow{5}{*}{21} & Maux de ventre, Parasitose & $\begin{array}{l}\text { Consommation directe des fruits et/ou macération aqueuse de la } \\
\text { racine ou de la poudre des feuilles pour boisson }\end{array}$ \\
\hline & & & Courbatures, Douleurs thoraciques & Infusion aqueuse des feuilles pour boisson et pour bain corporel \\
\hline & & & Maladies sournoises & Macération aqueuse des feuilles pour boisson \\
\hline & & & Enflure chez l'enfant & Macération aqueuse de la poudre des feuilles pour bain corporel \\
\hline & & & Paludisme & Macération aqueuse de l'écorce pour bain corporel \\
\hline \multirow[b]{2}{*}{ Khaya senegalensis } & \multirow[b]{2}{*}{11} & \multirow[b]{2}{*}{41} & Maux de ventre chez l'homme et le bétail & Macération aqueuse de l'écorce pour boisson \\
\hline & & & Démangeaisons, Boutons & $\begin{array}{l}\text { Macération aqueuse de l'écorce et/ou des feuilles pour boisson et } \\
\text { pour bain corporel }\end{array}$ \\
\hline \multirow{6}{*}{ Pterocarpu serinaceus } & \multirow{6}{*}{9} & \multirow{6}{*}{10} & Fracture & Infusion aqueuse de l'écorce pour boisson et pour bain corporel \\
\hline & & & Courbatures, Douleurs & Infusion aqueuse de l'écorce pour boisson et pour bain corporel \\
\hline & & & Anémie & Infusion aqueuse de l'écorce pour boisson et pour bain corporel \\
\hline & & & Maux de ventre & Macérationaqueusede l'écorce pour boisson \\
\hline & & & Toux & Macération aqueuse de l'écorce pour boisson \\
\hline & & & Rhume & Macération aqueuse de l'écorce pour boisson \\
\hline \multirow{4}{*}{ Vitex madiensis } & \multirow{4}{*}{8} & \multirow{4}{*}{19} & Blessures, Plaies de circoncision & Infusion aqueuse de l'écorce pour humectation \\
\hline & & & Démangeaisons, Boutons & Infusion aqueuse de l'écorce pour bain corporel \\
\hline & & & Maux de ventre & $\begin{array}{l}\text { Macération aqueuse de nouvelles feuilles (plus de l'arachide et du } \\
\text { sel) pour boisson }\end{array}$ \\
\hline & & & Vomissement & Macération aqueuse de l'écorce pour boisson \\
\hline \multirow{3}{*}{ Bambusa vulgaris } & \multirow{3}{*}{6} & \multirow{3}{*}{18} & Hyper/Hypotension & Infusion aqueuse des feuilles pour boisson \\
\hline & & & Stress, Peur, Agitation & Infusion aqueuse des feuilles pour boisson \\
\hline & & & Problèmes cardiaques & Macération aqueuse de l'écorce pour boisson \\
\hline
\end{tabular}




\begin{tabular}{|c|c|c|c|c|}
\hline ESPECES UTILES & $\begin{array}{l}\text { FMC } \\
(\%)\end{array}$ & $\mathrm{FL}(\%)$ & AFFECTIONS OU MAUX TRAITEES & PARTIES UTILISEES ET MODE D'EMPLOI \\
\hline & & & Hyper/ Hypoglycémie & Macération aqueuse de l'écorce pour boisson \\
\hline & & & Enflure de parties du corps & Macération aqueuse de l'écorce pour boisson \\
\hline \multirow{3}{*}{ Hexalobus monopetalus } & \multirow{3}{*}{6} & \multirow{3}{*}{12} & Maux de ventre & $\begin{array}{l}\text { Macération aqueuse de l'écorce pour boisson avec du lait caillé } \\
\text { et/ou mâchage directe de l'écorce }\end{array}$ \\
\hline & & & Fièvre jaune & Macération aqueuse de l'écorce pour boisson \\
\hline & & & Conjonctivite chez les bovins & $\begin{array}{l}\text { Rinçage des yeux du bovin avec une macération aqueuse de } \\
\text { l'écorce }\end{array}$ \\
\hline \multirow{4}{*}{ Adansonia digitata } & \multirow{4}{*}{6} & \multirow{4}{*}{8} & Enflure de parties du corps & Macération aqueuse de l'écorce pour boisson \\
\hline & & & Fièvre jaune & Macération aqueuse de l'écorce pour boisson \\
\hline & & & Maux de ventre & Sauce préparée avec les nouvelles feuilles à manger \\
\hline & & & Insuffisances pondérales infantiles & Feuillet d'écorce à attacher au cou et au pied de l'enfant \\
\hline \multirow{2}{*}{ Annona senegalensis } & \multirow{2}{*}{4} & \multirow{2}{*}{40} & Maux de ventre & Macération de l'écorce avec du lait caillé pour boisson \\
\hline & & & Sorts diaboliques & Macération aqueuse des feuilles pour bain corporel \\
\hline \multirow{3}{*}{ Ximenia americana } & \multirow{3}{*}{5} & \multirow{3}{*}{45} & Toux et vomissement & Macération aqueuse de la poudre des feuilles pour boisson \\
\hline & & & Douleurs corporelles & Infusion aqueuse des feuilles pour bain corporel \\
\hline & & & Infections buccodentaires & Macération aqueuse des feuilles pour bain de bouche \\
\hline \multirow[t]{2}{*}{ Tamarindus indica } & \multirow[t]{2}{*}{5} & \multirow[t]{2}{*}{14} & Maux des yeux & $\begin{array}{l}\text { Macération aqueuse de feuilles particulières (qui apparaissent } \\
\text { parfois) pour rinçage des yeux }\end{array}$ \\
\hline & & & Rhume & Consommation directe ou sous forme de jus des fruits \\
\hline \multirow[t]{2}{*}{ Detarium microcarpum } & \multirow[t]{2}{*}{5} & \multirow[t]{2}{*}{9} & Maux de ventre & $\begin{array}{l}\text { Consommation directe des fruits et/ou macération aqueuse de } \\
\text { l'écorce pour boisson }\end{array}$ \\
\hline & & & Diarrhée & Macération aqueuse de la poudre des noix pour boisson \\
\hline \multirow{4}{*}{ Lannea acida } & \multirow{4}{*}{5} & \multirow{4}{*}{7} & Dysenterie & Macération aqueuse de l'écorce pour boisson \\
\hline & & & Maux de ventre & Macération aqueuse de l'écorce pour boisson \\
\hline & & & Fièvre jaune & Macération aqueuse de l'écorce pour boisson \\
\hline & & & Maux des yeux & Macération aqueuse de l'écorce pour rinçage des yeux \\
\hline
\end{tabular}




\begin{tabular}{|c|c|c|c|c|}
\hline ESPECES UTILES & $\begin{array}{l}\text { FMC } \\
(\%)\end{array}$ & $\mathrm{FL}(\%)$ & AFFECTIONS OU MAUX TRAITEES & PARTIES UTILISEES ET MODE D'EMPLOI \\
\hline & & & Maux de dent & $\begin{array}{l}\text { Macération aqueuse de l'écorce pour bain de bouche et morceau } \\
\text { d'écorce à mâcher }\end{array}$ \\
\hline \multirow{3}{*}{ Balanites aegyptiaca } & \multirow{3}{*}{4} & \multirow{3}{*}{19} & Maux de ventre & Consommation directe des fruits \\
\hline & & & Hyper/Hypotension & Consommation directe des fruits \\
\hline & & & Parasitoses intestinales & $\begin{array}{l}\text { Macération aqueuse des feuilles pour boisson et consommation } \\
\text { directe des fruits }\end{array}$ \\
\hline \multirow{3}{*}{ Parkia biglobosa } & \multirow{3}{*}{4} & \multirow{3}{*}{16} & Fièvre & $\begin{array}{l}\text { Macération aqueuse de la poudre des pulpes et/ou des feuilles } \\
\text { pour boisson }\end{array}$ \\
\hline & & & Excès de sommeil & Macération aqueuse de l'écorce pour boisson \\
\hline & & & Constipation & Macération aqueuse de l'écorce pour boisson \\
\hline \multirow{3}{*}{ Stereospermum kunthianum } & \multirow{3}{*}{3} & \multirow{3}{*}{75} & Fièvre jaune & Macération aqueusede l'écorce pour boisson et/ou bain corporel \\
\hline & & & Crises épileptiques & Macération aqueuse de l'écorce pour bain corporel \\
\hline & & & Hyper/Hypotension & Macération aqueuse de l'écorce pour boisson \\
\hline \multirow{2}{*}{ Guiera senegalensis } & \multirow{2}{*}{2} & \multirow{2}{*}{67} & Incontinence urinaire & Décoction aqueuse des racines pour boisson \\
\hline & & & Maladies sournoises & Infusion aqueuse des feuilles pour boisson \\
\hline \multirow{2}{*}{ Nauclea latifolia } & \multirow{2}{*}{2} & \multirow{2}{*}{50} & Maux de ventre & Infusion aqueusedes feuilles et des racines pour boisson \\
\hline & & & Brûlures & Infusion aqueuse des feuilles pour bain corporel \\
\hline Pseudocedrela kotschyi & 2 & 50 & Poux, Démangeaisons, Boutons & $\begin{array}{l}\text { Infusion aqueuse de l'écorce pour bain corporel ou pour } \\
\text { humectation sur les plaies ou boutons. }\end{array}$ \\
\hline \multirow{2}{*}{ Mitragyna inermis } & \multirow{2}{*}{2} & \multirow{2}{*}{40} & Maux de ventre & Macération aqueuse de l'écorce pour boisson \\
\hline & & & Douleurs, Fatigue générale & Infusion aqueuse des feuilles pour boisson \\
\hline \multirow{2}{*}{ Terminalia macroptera } & \multirow{2}{*}{2} & \multirow{2}{*}{20} & Maux de ventre & Macération aqueuse de la racine pour boisson \\
\hline & & & Problèmes articulaires & Infusion aqueuse des feuilles pour bain corporel \\
\hline Acacia dudgeoni & 2 & 18 & Démangeaisons, Boutons & Macération aqueuse de l'écorce pour boisson et bain corporel \\
\hline \multirow{2}{*}{ Diospyrosmes piliformis } & \multirow{2}{*}{2} & \multirow{2}{*}{12} & Dysenterie & Infusion aqueuse des feuilles pour boisson \\
\hline & & & Maux de ventre chez l'enfant & Macération aqueuse de l'écorce pour boisson \\
\hline Ficus gnaphalocarpa & 2 & 8 & Aménorrhée & Infusion aqueuse de l'écorce pour boisson \\
\hline
\end{tabular}




\begin{tabular}{lccll}
\hline ESPECES UTILES & $\begin{array}{l}\text { FMC } \\
(\%)\end{array}$ & FL(\%) & AFFECTIONS OU MAUX TRAITEES & PARTIES UTILISEES ET MODE D'EMPLOI \\
\hline Cassia sieberiana & 1 & 50 & Maux de ventre & Infusion aqueuse des feuilles pour boisson \\
\hline Combretum micranthum & 1 & 50 & Hyper/Hypotension & Décoction aqueuse des feuilles pour boisson \\
\hline Gardeniae rubescens & 1 & 50 & Toux & Infusion aqueuse des feuilles pour boisson \\
\hline Prosopis africana & 1 & 50 & Rhume & Infusion aqueusedes feuilles pour boisson \\
\hline Afrormozia laxiflora & 1 & 33 & Arthrose & Infusion aqueuse des feuilles pour bain corporel \\
\hline Spondias mombin & 1 & 33 & Chaud pisse & Macération aqueuse des feuilles pour boisson \\
\hline Combretum glutinosum & 1 & 25 & Maux de ventre & Infusion aqueuse des feuilles pour boisson \\
\hline Terminalia avicennioides & 1 & 25 & Maux de ventre & Macération aqueuse de la racine pour boisson \\
\hline Ficus dicranostyla & 1 & 20 & Démangeaisons, Boutons & Macération aqueuse de l'écorce et des feuilles pour boisson \\
\hline Piliostigma reticulatum & 1 & 10 & Allergie & Macération aqueuse de l'écorce et de la racine pour boisson \\
\hline Cordia myxa & 1 & 4 & Problèmes cardiaques & Macération aqueuse de feuilles particulières (qui apparaissent \\
\hline
\end{tabular}


Affections traitées et espèces préférées : II ressort qu'au-delà d'affections plus ou moins banales telles que les démangeaisons, la fièvre, l'excès de sommeil, ou les allergies, les ligneux sont également utilisés pour traiter des maladies davantage préoccupantes, comme les crises épileptiques, l'hyper ou l'hypoglycémie, l'hyper ou l'hypotension, les problèmes cardiaques. Et les espèces ligneuses les plus utilisées à cet effet sont : Vitellaria paradoxa, citée par $31 \%$ des enquêtés, pour les multiples usages médicinaux de son beurre de karité ; Borassus aethoipum (18\%), surtout pour le traitement de la fièvre jaune ; Cordyla pinnata (15\%), particulièrement conte la diarrhée et les maux de ventre; Ziziphus mauritiana (15\%), notamment contre les vomissements; et Saba senegalensis $(12 \%)$, pour le traitement des courbatures et du paludisme entre autre. En plus d'elles, d'autres espèces, moins fréquemment indiquées, s'avèrent intéressantes en phytothérapie, au vu de leurs niveaux de fidélité. II s'agit de Khaya senegalensis, indiquée par $11 \%$ des enquêtés, avec $41 \%$ de fidélité à la phytothérapie, essentiellement contre les dermatoses et les maux de ventre chez l'homme et le bétail ; et Stereospermum kanthianum, citée à seulement $3 \%$, mais avec un niveau de fidélité de $75 \%$, contre les crises épileptiques et l'hyper/hypotension.

Parties ligneuses utilisées en phytothérapie : Une analyse secondaire du tableau 2 a permis d'établir les fréquences d'implication des différentes parties ligneuses dans les usages phytothérapiques (figure 2).

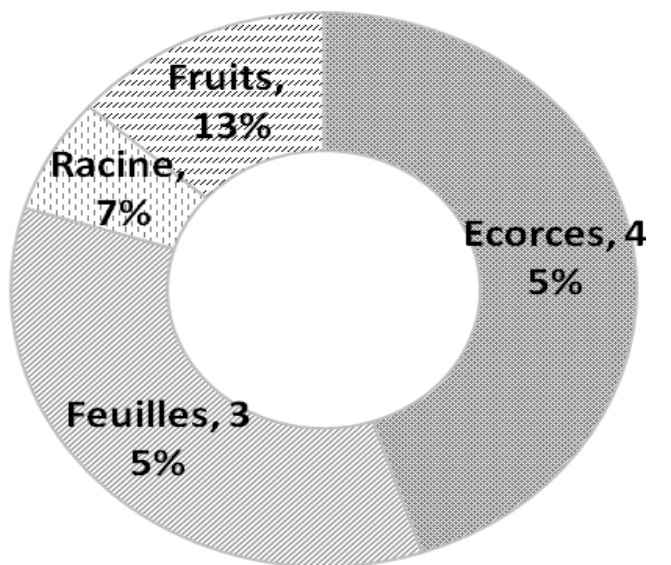

Figure 2 : Fréquence d'indication des parties ligneuses en phytothérapie.

II apparait que les écorces et les feuilles, impliquées respectivement dans $45 \%$ et $35 \%$ des traitements, sont les parties des ligneux les plus utilisées en phytothérapie. Les racines, bien qu'utilisées uniquement en pharmacopée, restent les parties des ligneux les moins impliquées (7\%). Même les fruits ont été plus fréquemment mentionnés dans des usages médicinaux (13\%). Bien qu'il s'avère quelque peu difficile de faire la part des choses entre les rôles alimentaire et thérapeutique des fruits. D'autant que pour un traitement donné, ils sont généralement utilisés en association avec les écorces, les feuilles ou les racines et sont souvent consommés directement, comme le montre, plus en détail, l'analyse sur les modes d'administration.

Mode de préparation des parties ligneuses utilisées: Les parties ligneuses impliquées en phytothérapie font globalement l'objet de cinq formes de préparations: la macération, la décoction, l'infusion, le rinçage et le broyage. La macération désigne un procédé (ainsi que le liquide qui en résulte) consistant à laisser séjourner un solide dans un liquide "froid" pour en extraire les composés solubles, ou bien pour qu'il absorbe de ce liquide afin d'en obtenir le parfum ou la saveur. Elle est qualifiée d'aqueuse quand le liquide est de l'eau. La macération diffère de l'infusion, où un solide est plongé dans un liquide initialement bouillant qu'on laisse refroidir, et de la décoction, où le liquide est maintenu bouillant avec le solide. Le rinçage diffère de ces procédés par son bref temps de contact avec le liquide, dus à son objectif qui est juste de nettoyer le solide, sans extraire ses composés solubles. Le broyage, quant à lui, consiste à réduire une matière "sèche" à une dimension donnée, correspondant à son emploi, ou facilitant la séparation de constituants hétérogènes. Pour une matière "fraiche", il s'agit d'une mise en dispersion, par action mécanique, de pigments et de matières de charge dans un liant en vue d'obtenir une pâte. (Dictionnaire de l'Académie française, 2005 et Dictionnaire de la langue française, Larousse, 1989). La figure 3 présente une analyse croisée des techniques préparatoires et des parties des ligneux impliquées en pharmacopée. 


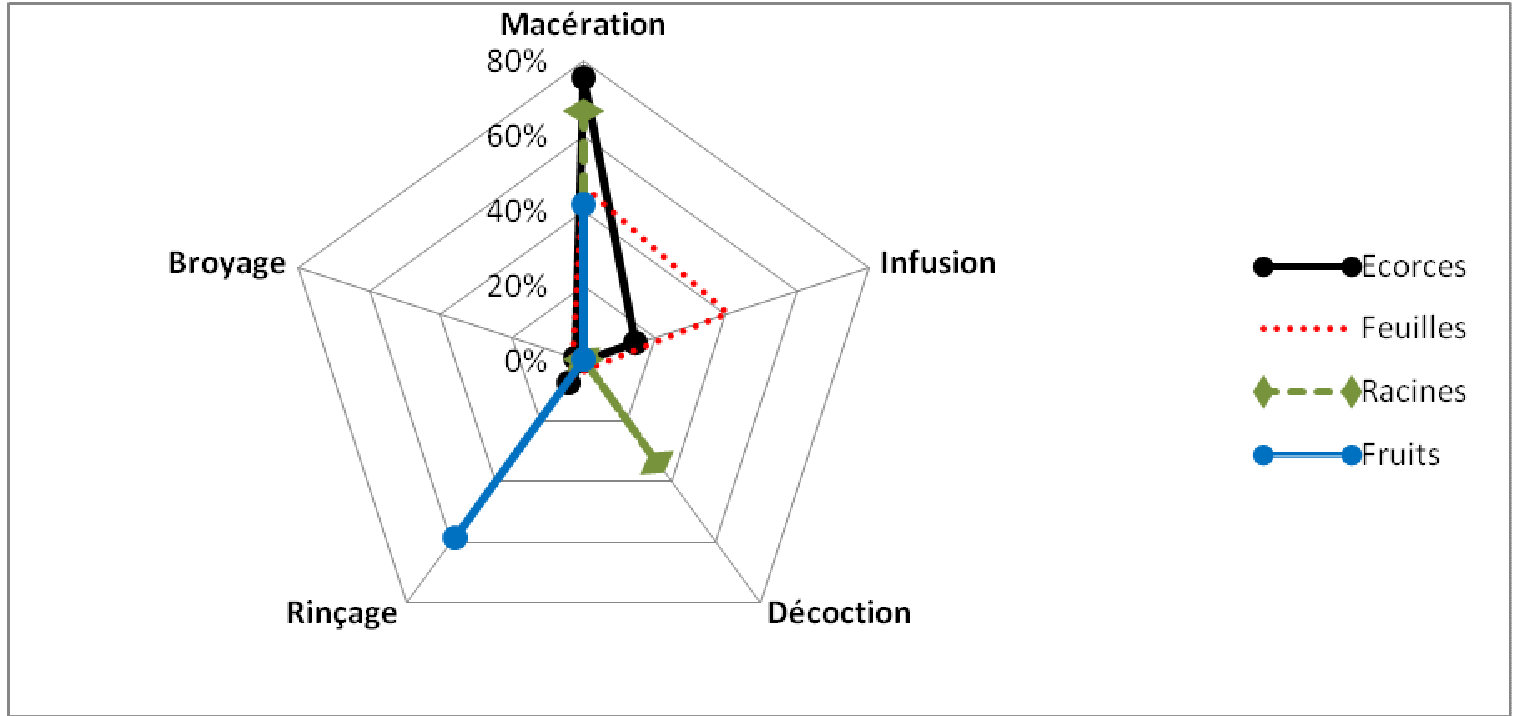

Figure 3 : Fréquence des modes de préparation selon les parties des ligneux en phytothérapie.

De toutes les parties des ligneux utilisées en phytothérapie, seules les feuilles font l'objet, à la fois, des 5 modes de préparation identifiées, quoique la décoction (3\%), le broyage après séchage (3\%) et le rinçage rapide $(6 \%)$ soient relativement rares. En fait, la macération $(47 \%)$ et l'infusion $(41 \%)$ constituent les formes de préparation des feuilles les plus communes. Alors que, les écorces sont principalement macérées $(76 \%)$, parfois infusées $(15 \%)$ ou rincées $(7 \%)$, rarement broyées après séchage $(2 \%)$, mais jamais décoctées. Cette absence de décoction est également notée pour les fruits, qui en plus ne font pas l'objet de d'infusion. Ils sont très essentiellement rincés (58\%) ou macérés $(42 \%)$. Alors que pour les racines, la macération $(67 \%)$ et la décoction $(33 \%)$ constituent les seules formes de préparation pour l'usage thérapeutique. Ainsi globalement, la macération s'avère être la forme de préparation la plus commune $(60 \%)$, suivie de l'infusion $(21 \%)$, puis du rinçage $(13 \%)$. La décoction $(3 \%)$ et le broyage après séchage $(2 \%)$ étant les formes de préparation les moins fréquentes. Formes d'administration des parties des ligneux: La figure 4 montre les relations entre les modes préparatoires et les formes d'administration de leurs produits :

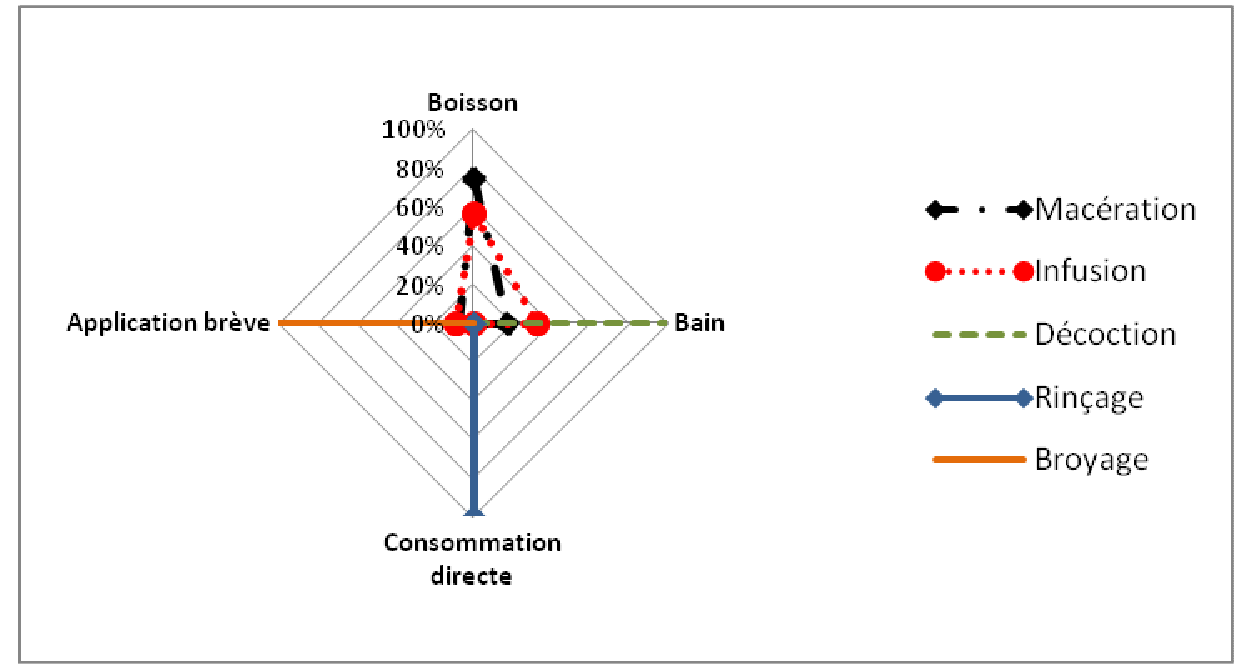

Figure 4 : Fréquence des formes d'administration selon les modes de préparation

Il apparait que les macérations sont principalement destinées à la boisson (75\%), au bain corporel (18\%) et plus rarement à une brève forme d'application $(7 \%)$, comme le rinçage des yeux ou de la bouche ou
I'humectation de surfaces cutanées affectées. De même, les produits d'infusion sont souvent bus $(53 \%)$, parfois utilisés pour le bain $(33 \%)$, ou plus rarement pour une brève application (10\%). Par 
contre, les produits de toutes les autres formes de préparations sont dédiés à des formes spécifiques d'administration : En effet, les parties décoctées sont destinées exclusivement au bain, celles rincées à la consommation directe et celles broyées à une brève application.

Pratiques mystiques et savoirs ésotériques liés à la phytothérapie: II convient, toutefois de mentionner qu'au-delà de ces formes de préparation et d'administration classiques, la pharmacopée malinké, implique parfois des pratiques mystiques nécessitant un certain savoir ésotérique. Ainsi, pour certains traitements, soit ce sont les feuilles disposées à l'envers sur le sol qui doivent être utilisées, soit certaines feuilles spéciales qui apparaissent occasionnellement qui sont ciblées, soit il y'a des jours ou des heures spécifiques durant lesquels les parties de l'arbre doivent être prélevées, soit il y a des formules consacrées à réciter au moment du prélèvement, de la préparation et/ou de l'administration du produit. Et ces pratiques complémentaires restent l'apanage des populations, qui les mentionnent au passage, sans vouloir les expliciter en détail.

Niveau de consensus autour des usages phytothérapiques: A partir de l'ensemble des citations, nous avons identifié 18 grandes catégories d'usages phytothérapiques, dont les nombres d'indication d'usage, les nombres d'espèces impliquées et les niveaux de consensus informateurs sont présentés dans le tableau 3 .

Tableau 3: Catégories de traitements phytothérapiques, Affections concernées, Nombres d'indications, Nombres d'espèces et Facteurs de consensus Informateurs.

\begin{tabular}{|c|c|c|c|c|}
\hline Catégorie de traitement & Affections concernées & N. ind. & N. esp. & $F_{i c}$ \\
\hline Psycho-émotionnel & Stress, Peur, Agitation & 3 & 1 & 1.00 \\
\hline Paludique & Paludisme & 2 & 1 & 1.00 \\
\hline Asthénique & $\begin{array}{l}\text { Fatigue, courbature, Faiblesse, } \\
\text { paresse }\end{array}$ & 13 & 3 & 0.83 \\
\hline Nausées/Vomissements & Nausées, Vomissement & 12 & 3 & 0.82 \\
\hline Fièvre jaune & Fièvre jaune & 33 & 7 & 0.81 \\
\hline Respiratoire & Rhume, Toux, Tuberculose & 19 & 8 & 0.61 \\
\hline Gastro-intestinal & $\begin{array}{l}\text { Maux de ventre, diarrhée, } \\
\text { constipation, dysenterie, parasitose }\end{array}$ & 42 & 17 & 0.61 \\
\hline Cardiovasculaire & $\begin{array}{l}\text { Problème cardiaque, Hypo } \\
\text { /Hypertension }\end{array}$ & 11 & 5 & 0.60 \\
\hline Ophtalmologique & Maux des yeux, Conjonctivite & 7 & 4 & 0.50 \\
\hline Dermatologique & $\begin{array}{l}\text { Démangeaison, bouton, enflure, } \\
\text { brûlures plaies allergie }\end{array}$ & 19 & 11 & 0.44 \\
\hline Osseuse/Articulaire & Fracture, arthrose & 4 & 3 & 0.33 \\
\hline Pédiatrique & $\begin{array}{l}\text { Apparition des dents, insuffisance } \\
\text { pondérale, maux de ventre chez } \\
\text { l'enfant }\end{array}$ & 8 & 6 & 0.29 \\
\hline Sournois/Mystique & $\begin{array}{l}\text { Mauvais diabolique, maladies } \\
\text { sournoises }\end{array}$ & 15 & 11 & 0.29 \\
\hline Glycémique/Diabétique & Hypo / Hyperglycémie, Diabète & 2 & 2 & 0.00 \\
\hline Sexuel/Gynécologique & $\begin{array}{l}\text { Chaud pisse, Aménorrhée, } \\
\text { Incontinence urinaire }\end{array}$ & 5 & 5 & 0.00 \\
\hline Fièvre & Fièvre & 2 & 2 & 0.00 \\
\hline Mental/comportemental & $\begin{array}{l}\text { Crise épileptique, Excès de } \\
\text { sommeil }\end{array}$ & 2 & 2 & 0.00 \\
\hline Bucco-dentaire & $\begin{array}{l}\text { Maux de dent, Infections bucco } \\
\text { dentaires }\end{array}$ & 2 & 2 & 0.00 \\
\hline
\end{tabular}

$\mathrm{N}$. ind. = Nombre d'indication d'usage $; \mathrm{N}$. esp. = Nombre d'espèces impliquées dans cette catégorie de traitement. 
Seuls les traitements d'affections ou de maux psycho-émotionnels et paludiques ont fait l'objet d'un maximum de consensus (100\%), avec l'usage exclusif et respectif de Bambusa vulgaris et Saba senegalensis. L'asthénie a enregistré un niveau de consensus assez élevé (83\%), dû essentiellement à Vitellaria paradoxa (utilisée dans $85 \%$ des traitements antiasthéniques). Les traitements des nausées/vomissements (82\%) et de la fièvre jaune $(81 \%)$ ont présenté aussi des facteurs de consensus relativement élevés. Ces niveaux de consensus s'expliquent respectivement par Ziziphus mauritiana

\section{DISCUSSION}

A l'instar de nombreuses communautés traditionnelles d'Afrique (Sarr et al., 2013; Cheikhyoussef et al., 2011), d'Amérique latine (Caceres et al., 1987; Argueta et Cano, 1994 ; Canales et al., 2005) et d'Asie (Srinivasan et al., 2001; Anaïs, 2013), les malinkés du terroir de Khossanto disposent d'une pharmacopée assez diversifiée, impliquant un cortège floristique ligneux fort : 40 espèces relevant de 37 genres et inféodés à 21 familles. Cette flore phytothérapique implique l'essentiel des espèces ligneuses utiles de la localité (Gning et al., 2013). D'un point de vue taxonomique, les Caesalpiniaceae et les Combretaceae, qui sont les familles les plus importantes en phytothérapie, figurent par ailleurs parmi les espèces les mieux représentées dans la localité. Ce qui corrobore les propos de Moerman et al. (1999), qui affirment que les familles floristiques les mieux représentées dans une région sont souvent les plus utilisées. Au-delà de la diversité des espèces phytothérapiques, la richesse de la pharmacopée malinké apparait également à travers la proportion des populations disposant de savoirs et savoir-faire phytothérapiques $(76 \%$ des enquêtés reconnaissent des vertus médicinaux à $87 \%$ des ligneux utiles de la localité), l'étendu du spectre pathologique pris en charge (une quarantaine d'affections et de maux traités) ainsi que l'importance (201 expressions d'usage) et la précision traitements décrits (parties utilisées, modes de préparation et d'administration). Par ailleurs, ce rôle central des plantes dans la santé des populations se trouve fortement entretenu la rareté des structures sanitaires au niveau de la localité (avec seulement le centre de santé de Khossantovillage et la maternité de Brassan pour toute la communauté rurale). Ainsi, les populations recourent naturellement aux plantes qui sont depuis toujours une source habituelle de remèdes sous forme de préparations traditionnelles ou de principes actifs purs (Farnsworth et al. 1986). Cette important rôle médicinal de l'arbre, découvert de manière empirique (utilisée dans $85 \%$ des traitements contre les nausées et vomissements) et Borassus aethiopum (indiquée dans $82 \%$ des traitements contre la fièvre jaune). Par ailleurs, les affections gastro-intestinales (42\% d'indications de traitement), respiratoires $(19 \%)$, dermatologiques (19\%), sournoises/mystiques $(15 \%)$ et cardiovasculaires (11\%) ont aussi fréquemment traitées avec les ligneux. Mais, elles présentent des niveaux de consensus moins consistants (entre 61 et $29 \%$ ), dus au nombre important des espèces (8 à 17) impliquées dans leurs traitements.

et transmise de façon traditionnelle à travers les générations, semble être confirmé par recherches scientifiques et applications pharmaceutiques. Par exemple, en 1986, environ 119 substances chimiques obtenues à partir de 91 espèces végétales étaient utilisées comme des médicaments importants dans 62 classes thérapeutiques (Farnsworth et al.1986). En 1995, les 25 produits pharmaceutiques les mieux vendus dans le monde, 12 étaient d'origine naturelle végétale (Baker et al., 1995). Toutefois, I'OMS (2012) souligne que "leur innocuité, leur efficacité et leur qualité dépendent de la qualité des matières premières dont ils sont tirés et de la manière dont les éléments sont manipulés pendant le processus de production".C'est dans ce sens que les modes de préparation et d'administration des parties ligneuses phytothérapiques ont été aussi étudiés. A ce niveau, il est apparu, qu'à l'image des guérisseurs traditionnels de Oshikoto en Namibie (Cheikhyoussef, 2011) et des populations de Lour Escale et Ida Mouride (Sarr, 2013), les malinkés de Khossanto utilisent le plus souvent les écorces et les feuilles des ligneux pour les traitements. Et ce sont les décoctions, macérations, infusions et produits de rinçage de ces parties ligneuses qui sont généralement, bus, directement consommés, utilisés pour le bain ou de brèves applications. Mais, en plus de ces prescriptions explicites, la pharmacopée malinké implique parfois des pratiques mystiques nécessitant un certains savoirs ésotériques, restent l'apanage des populations. En effet, les traitements sont souvent accompagnés de pratiques plus ou moins occultes, qu'elles mentionnent au passage, sans vouloir les expliciter en détail. Cette situation est aussi soulignée par AYENSU (1983), qui affirme que "dans la culture africaine, les médecins traditionnels sont toujours considérés en même temps comme des chefs spirituels influents, mêlant la magie et la religion à la thérapeutique. La maladie est combattue par les pouvoirs spirituels occultes de l'homme aussi bien que par l'administration de plantes auxquelles 
on a reconnu des vertus curatives particulières". En fin, les Facteurs de Consensus Informateurs ont montré des forts niveaux de consensus des populations au tour des traitements contre des affections psycho-émotionnelles (100\%), paludiques $(100 \%)$, asthéniques $(83 \%)$, contre les nausées/vomissements $(82 \%)$ et contre la fièvre jaune (81\%). Ainsi, Bambusa vulgaris, Saba senegalensis, Vitellaria paradoxa, Ziziphus mauritiana et Borassus aethiopum, respectivement

\section{CONCLUSION}

Cette étude a mis en évidence la place centrale de l'arbre dans la santé des populations de la communauté rurale de Khossanto, à travers la diversité floristique de la pharmacopée malinké (40 d'espèces ligneuses, de 37 genres et de 21 familles botaniques impliquées) et la richesse des savoirs et savoir-faire locaux phytothérapiques (201 expressions d'usage traitant une quarantaine d'affections, à travers presque toutes les parties ligneuses, dont les modes de préparation et d'administration sont décrites de manière assez explicite). Toutefois, des certaines pratiques, mystiques associées à cette phytothérapie, la rendent quelque peu ésotérique. En plus, la qualité

\section{REFERENCES BIBLIOGRAPHIQUES}

Argueta, V. A., Cano A. J., 1994. Atlas de las Plantas de la Medicina Tradicional Mexicana. Instituto Nacional Indigenista. Mexico. p. 1785.

Caceres A., Giron, L.M., Alvarado, S.R., Torres, M.F., 1987. Screening of antimicrobial activity of plants populary used in Guatemala for the treatment of dermatomucosal diseases. Journal of Ethnopharmacology 20, 223-237.

Camus H. et DebuissonJ., 1964. Étude hydrogéologique des terrains anciens $d u$ Sénégal Oriental. Campagne 1962-1963. Rapp. B.R.G.M., Dakar, Dak. 64-06, 143 p.

Canales M., HermandesT., Caballero J., Romo De Vivar A., AvilaG., Duran A.,Lira R., 2005. Informant consensus factor and antibacterial activity of the medicinal plants used by the people of San Rafael coxcatlan. Puebla, Mexico, Journal of Ethnopharmacology, 97 : 429-439.

Cheikhyoussef A., Ashekele H., Shapi M.,Matengu K., 2011. Ethnobotanical study of indigenus knowledge on medicinal plant use by traditional healers in Oshikoto region, Namibia.Journal of Ethnobiology and Ethnomedicine. 7-10. responsables des niveaux de consensus dans ces catégories, seraient intéressantes pour la recherche d'éléments bioactifs. En effet, une plante utilisée par les populations de manière répétitive pour un traitement s'avère généralement biologiquement active (Trotter et al., 1986). D'ailleurs, selon King et al. (1996), les recherches de bio-activités médicales sont positives à au moins $50 \%$ chez des plantes révélées intéressantes par des méthodes ethnobotaniques.

des parties ligneuses utilisées, ainsi que les procédés de préparation et d'administration déterminent en grande partie leurs innocuités et leurs efficacités. D'où la nécessité, d'étudier plus spécifiquement les éléments responsables de ces vertus thérapeutiques, surtout chez certaines espèces qui ont fait l'objet de forts consensus pour leurs efficacités contre des traitements spécifiques: Bambusa vulgaris (affections psycho-émotionnelles), Saba senegalensis (paludisme), Vitellaria paradoxa (asthénie), Ziziphus mauritiana (les nausées/vomissements) et Borassus aethiopum (la fièvre jaune).

Diouf S., 1999. Hydrogéologie en zone de socle cristallin et cristallophyllien du Sénégal oriental. Doctorat de 3ème Cycle en hydrogéologie. FST. UCAD, 86p.

Farnsworth N. R., Akerele O., Bingel A. S., Soejarto D. D., Guo Z., 1986. Place des plantes médicinales dans la thérapeutique. Bulletin de l'OMS ; 64 (2) : 159-175.

Gning O. N., SARR O., GUEYE M., AKPO L. E., NDIAYE P. M., 2013. Valeur socioéconomique de l'arbre en milieu malinké (Khossanto, Sénégal).Journal of Applied Biosciences $70: 5617-5631$

King, S.R., Carlson, T.J., Moran, K., 1996. Biological diversity, indigenous knowledge, drug discovery and intellectual property rights: Creating reciprocity maintaining relationships. Journal of Ethnopharmacology 51(1-3) : 45-57.

Lefébure A., 2013, Les grands principes de la médecine traditionnelle chinoise, article, JOL Presse

Moerman D. E., Pemberton R.W., Kiefer D., Berlin B., 1999.A comparative analysis of five medicinal floras. Journal of Ethnopharmacology 19(1) : 49-67. 
Organisation Mondiale de la Santé (OMS)., 2012. Médecine Traditionnelle, Aide-mémoire $\mathrm{N}^{\circ} 134$, Centre des Médias de l'OMS, http://www.who.int/mediacentre/factsheets/fs 134/fr/index.html

Sarr O., 2013. L'arbre en milieu soudano-sahélien dans le bassin arachidier (Centre-Sénégal). Journal of Applied Biosciences 61: 4515 4529.

Srinivasan D., Sangeetha N., Suresh T., Lakshmana P.P., 2001. Antimicrobial activity of certain Indian medicianal plants used in folkloric medicine. Journal of Ethnopharmacology 74(3), 217-220.

Trotter R.T., Logan M.H., 1986. Informants consensus : a new approach for identifying potentially effective medicinal plants.In Plants in Indigenous Medicine and Diet. Edited by: Etkin NL. Redgrave Publishing Company, Bedford Hill, NY ; pp. :91-112.

Vaney T., 2013." La phytothérapie"; Article sur "Les thérapies naturelles".

http://www.vaneytherapies.ch/phytotherapie.html

Weller S.C., Romney A.K., 1998. Systematic Data Collection. Qualitative Research Methods Series, 10.SAGE. Publications, Newbury Park, USA. 